\title{
Turbo Equalization For Space-Time Block Coded FWA Systems
}

\author{
Pei Xiao, Rolando Carrasco \\ School of EECE, University of Newcastle \\ Newcastle, NE1 7RU, United Kingdom \\ E-mail: pei.xiao@ncl.ac.uk, \\ r.carrasco@ncl.ac.uk
}

\begin{abstract}
The use of multiple antennas in combination with advanced detection techniques, such as turbo equalization is an effective means for a Fixed Wireless Access (FWA) system to provide high quality and high data rate services. Alamouti's space-time block code (STBC) with two transmit antennas and one or two receive antennas over frequency selective FWA SUI-3 channels is considered in this paper. We propose a turbo equalization algorithm that aims at exploiting the multipath diversity and reducing the effect of intersymbol interference (ISI), and in the meantime, keeping the desired feature of the original Alamouti detection algorithm, i.e, achieving spatial diversity with simple linear processing.
\end{abstract}

\section{INTRODUCTION}

Fixed wireless access (FWA) is quickly emerging as a significant network access alternative for the delivery of voice, data, Internet, video and multimedia type applications to business and residential customers. FWA systems offer a very cost-effective way of building an access network. Easy maintainability, low incremental costs and portability are key benefits of this wireless alternative [1]. Standardization of FWA systems is currently being undertaken by the IEEE 802.16 group [2] and the ETSI HIPERMAN group [3]. Both orthogonal frequency division multiplexing (OFDM) and single-carrier solutions have been adopted in IEEE 802.16 standard as two alternatives for FWA systems operating at 2-11 GHz bands [4].

The major challenge of designing a FWA system is to provide high data rate wireless access with wire-line quality. The high requirement for quality arises because FWA systems have to compete with cable modems and digital subscriber line (DSL) approaches which operate over fixed channels and hence are able to provide very good quality. In recent years, space-time coding has emerged as one of the most promising technologies for meeting the high data rate and high service quality requirements. There are two main types of space-time codes, namely, space-time trellis codes (STTC) [5] and space-time block codes (STBC) [6,7]. The performance of STTC and STBC was compared in [8]. The advantage of STTCs over STBCs is the provision of coding gain, however, this is achieved at the cost of high complexity encoders and decoders. For the purpose of this work, we consider the use of STBC, in particular, the two-antenna transmit diversity scheme [6] proposed by Alamouti.

At high data rates, the intersymbol interference (ISI) introduced by frequency-selective fading channels becomes a

\author{
Ian Wassell \\ Computer Laboratory \\ University of Cambridge \\ Cambridge, CB3 0FD, United Kingdom \\ E-mail: ijw24@eng.cam.ac.uk
}

severe problem. The key building block in combating ISI is the equalizer, or more effectively, the turbo equalizer that performs equalization and channel decoding jointly in an iterative manner. The existing techniques can be broadly classified into trellis (MAP) based approach [9] and linear MMSE-filter based approach $[10,11]$. In [12], the trellis based approach is applied to STTC coded multiple-input, multiple output (MIMO) systems, and channel shortening technique (leading to a smaller number of trellis states) is proposed to reduce the complexity of the space-time turbo equalization. The filter-based approach proposed in $[10,11]$ for single-input, single-output channels is extended to MIMO systems, e.g., in [13-15]. However, the MMSE equalizers introduced in $[13,14]$ are applied to the MIMO systems without STBC or STTC. Moreover, only a single-input, multiple-output system (for a single user) is considered in [14]. The turbo multiuser detection technique previously proposed in [10] is extended to STBC and STTC coded systems in [15-17] for flat-fading channels and frequency-seletive fading channels, respectively. In [18], the STBC originally developed for flat fading channels is generalized for frequency selective channels, where the transmitted signals are coded on a block-to-block basis rather than a symbol-by-symbol basis. This is so-called time-reversal (TR) STBC. However, TR-STBC only converts a multipleinput, multiple-output (MIMO) channel into a single-input, single-output (SISO) channel, it does not, however, eliminates ISI to which conventional SISO equalization has to be applied. In this paper, we introduce a different approach which aims at fully exploiting the spatial and temporal diversities for the STBC coded systems over frequency selective channels, and compare its performance with the TRSTBC approach as well as the conventional MMSE filter based turbo equalization. We use the FWA systems as an example. However, the proposed turbo equalization scheme can be easily extended to other STBC coded systems.

\section{System Model}

Fig. 1 shows the baseband representation of the two branch transmit diversity scheme under study. The information sequence $\left\{b_{n}\right\}$ is convolutionally encoded into coded bits $\left\{u_{n}\right\}$, which are subsequently interleaved and each block of two coded and interleaved bits $u_{n}^{\prime}[0], u_{n}^{\prime}[1]$ is mapped into one of the four QPSK symbols. The interleaver and deinterleaver are denoted as $\Pi$ and $\Pi^{-1}$, in Fig. 1 and Fig. 2, respectively.

We use the space-time coding scheme proposed in [6]. The encoding and transmission sequence of the QPSK symbols are shown in Table 1. The transmitted symbols are 


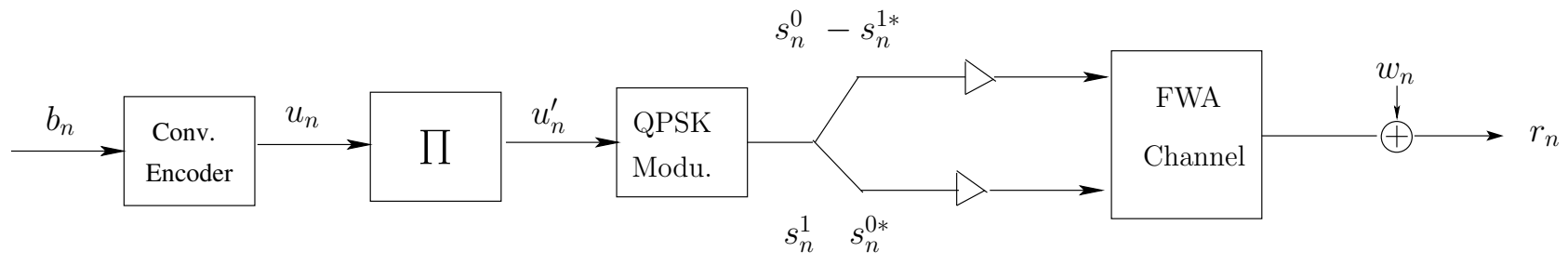

Figure 1: Diagram for the STBC FWA system with 2TX-1RX antennas.

grouped into blocks of 2 symbols at each antenna. At a given time, two symbols are simultaneously transmitted from two antennas. At time instance $t$, the symbol transmitted from the first antenna is denoted as $s_{n}^{0}$, and the symbol transmitted from the second antenna is denoted as $s_{n}^{1}$. During the next symbol period $t+T$, symbol $-s_{n}^{1 *}$ is transmitted from the first antenna, and $s_{n}^{0 *}$ is transmitted from the second antenna, where ${ }^{*}$ denotes the conjugate transpose operation when applied to matrices and vectors, and simply the conjugate when applied to scalars.

For simplicity, we assume two transmit antennas and one receive antenna in the derivation of the proposed turbo equalization algorithm. However, its extension to systems with multiple receive antennas is straightforward. Each complex channel coefficient is denoted as $h_{i j}^{l}$ where the first (second) subscript $i(j)$ is the index of the transmit (receive) antenna, the superscript $l$ refers to the number of the channel tap. For example, $h_{10}^{0}$ denotes the channel coefficient corresponding to the first tap of the channel between tx1 and $\mathrm{rx} 0$.

Tailored for different terrain conditions, a set of 6 typical channel models, known as the Stanford University Interim (SUI) Channel Models have been proposed in [19] for simulation, design, development and testing of FWA systems. For the purpose of this study, we select the SUI-3 channel which has 3 taps with a tap spacing of 500ns, and maximum tap delay of 1000ns. The channel coefficients are complex Gaussian random variables and are assumed to remain constant during the transmission of one block of data. The amplitude of the first tap $\left|h_{i j}^{0}\right|$ is characterized by a Ricean distribution due to the presence of line of sight propagation. The amplitudes of the other two taps $\left|h_{i j}^{1}\right|,\left|h_{i j}^{2}\right|$ are Rayleigh distributed. For simplicity, we assume the transmitted data rate is $4 \mathrm{Mbps}$ with QPSK modulation so that the multipath fading is modeled as a tapped-delay line with adjacent taps spaced equally at the symbol duration. The received signals at antenna rx0 during the two symbol periods $t$ and $t+T$ can be formed as

$$
\begin{gathered}
r_{n}^{0}=h_{00}^{2} s_{n-1}^{0}-h_{00}^{1} s_{n-1}^{1 *}+\underline{h_{00}^{0} s_{n}^{0}}+h_{10}^{2} s_{n-1}^{1} \\
+h_{10}^{1} s_{n-1}^{0 *}+\underline{h_{10}^{0} s_{n}^{1}}+w_{n}^{0}
\end{gathered}
$$

Table 1: The encoding and transmission sequence for the two transmit antenna system.

\begin{tabular}{|c|c|c|c|c|c|c|}
\hline \hline & $t-2 T$ & $t-T$ & $t$ & $t+T$ & $t+2 T$ & $t+3 T$ \\
\hline tx0 & $s_{n-1}^{0}$ & $-s_{n-1}^{1 *}$ & $s_{n}^{0}$ & $-s_{n}^{1 *}$ & $s_{n+1}^{0}$ & $-s_{n+1}^{1 *}$ \\
\hline tx1 & $s_{n-1}^{1}$ & $s_{n-1}^{0 *}$ & $s_{n}^{1}$ & $s_{n}^{0 *}$ & $s_{n+1}^{1}$ & $s_{n+1}^{0 *}$ \\
\hline
\end{tabular}

$$
\begin{aligned}
r_{n}^{1}=- & h_{00}^{2} s_{n-1}^{1 *}+h_{00}^{1} s_{n}^{0}-\underline{h_{00}^{0} s_{n}^{1 *}}+h_{10}^{2} s_{n-1}^{0 *} \\
& +h_{10}^{1} s_{n}^{1}+\underline{h_{10}^{0} s_{n}^{0 *}}+w_{n}^{1} .
\end{aligned}
$$

where $w_{n}^{0}, w_{n}^{1}$ are the complex additive white Gaussian noise with zero mean and variance $N_{0}$. The desired symbols in the above equations are underlined so that they can be distinguished from the interference symbols and the noise.

\section{TURBo EQUALIZATION WITH MODIFIED Alamouti AlgORITHM}

The task of the receiver is to detect the transmitted information bits $\left\{b_{n}\right\}$ given the received observations $\left\{r_{n}^{0}, r_{n}^{1}\right\}$. To this end, we need first to detect the transmitted QPSK symbols $\left\{s_{n}^{0}, s_{n}^{1}\right\}$ which are corrupted with ISI and AWGN noise. An equalizer is required to remove the detrimental effect of ISI. The estimated symbols are then converted to coded bits, which are subsequently deinterleaved and decoded to obtain an estimate of the information sequence. Here, we focus on the turbo equalization algorithm which combines equalizer and channel decoder in an iterative fashion.

The proposed turbo equalization algorithm is illustrated in Fig. 2. First, we use a training sequence to acquire a channel estimate $\hat{h}_{i j}^{l}$ using some channel estimation algorithm. In the meantime, a modified Alamouti algorithm is used to obtain the soft values of the transmitted symbols in the form of log-likelihood ratio (LLR) $\left\{\lambda\left(s_{n}\right)=\right.$ $\left.\lambda\left(x_{n}\right)+j \lambda\left(y_{n}\right)\right\}$ where $s_{n}$ denotes either $s_{n}^{0}$ or $s_{n}^{1}$. The channel estimate $\hat{h}_{i j}^{l}$ and symbol estimates $\left\{\lambda\left(s_{n}\right)\right\}$ are passed to the equalizer, which computes $\widetilde{s_{n}}$, the soft decision of $s_{n}$. The soft estimate of the symbol is then mapped to the LLR values of coded bits $\left\{\lambda\left(u_{n}^{\prime} ; O\right)\right\}$ by the symbol-to-bit converter (SBC), which are deinterleaved to yield $\left\{\lambda\left(u_{n} ; I\right)\right\}$. Based on the soft inputs, a Log-MAP decoder computes the LLR for each information bit $\lambda\left(b_{n} ; O\right)$ and each coded bit $\lambda\left(u_{n} ; O\right)$. The former is used to make decisions on the transmitted information bit at the final iteration, and $\lambda\left(u_{n} ; O\right)$ is interleaved and passed through a bit-to-symbol converter (BSC) to derive a soft symbol estimate $\lambda\left(s_{n}\right)$, which is used for equalization at the next iteration. We use the notations $\lambda(\cdot ; I)$ and $\lambda(\cdot ; O)$ to denote the input and output ports of a soft-input and soft-output device. The equalization algorithm will be described in detail next.

\section{A. Equalizer design at the first non-cancellation stage}

For the received signals expressed by (1), a direct implementation of the Alamouti algorithm yields

$$
\begin{aligned}
& \widetilde{s_{n}^{0}}=\hat{h}_{00}^{0 *} r_{n}^{0}+\hat{h}_{10}^{0} r_{n}^{1 *}=\left(\hat{h}_{00}^{0 *} h_{00}^{0}+\hat{h}_{10}^{0 *} h_{10}^{0}\right) s_{n}^{0}+\eta_{n}^{0} ; \\
& \widetilde{s_{n}^{1}}=\hat{h}_{10}^{0 *} r_{n}^{0}-\hat{h}_{00}^{0} r_{n}^{1 *}=\left(\hat{h}_{00}^{0 *} h_{00}^{0}+\hat{h}_{10}^{0 *} h_{10}^{0}\right) s_{n}^{1}+\eta_{n}^{1},
\end{aligned}
$$




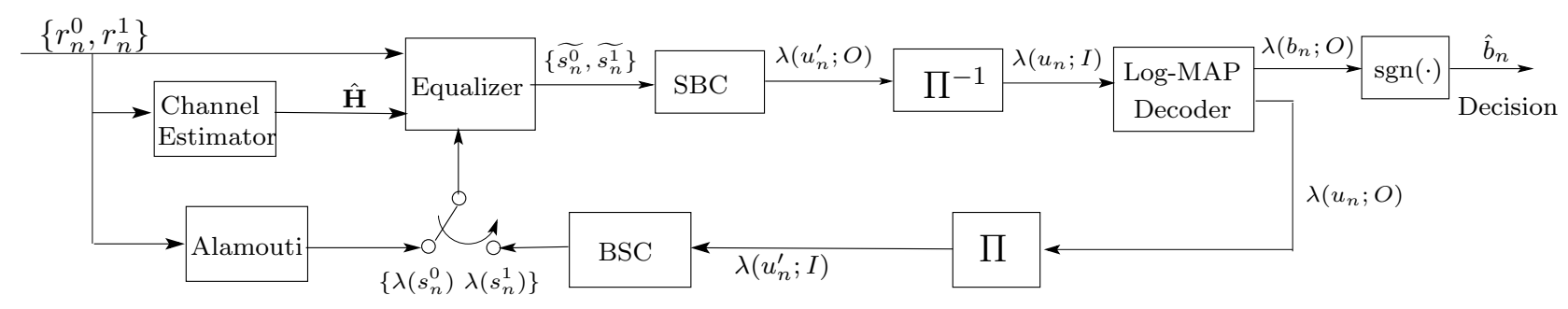

Figure 2: Turbo equalization for the FWA SUI-3 channel.

where $\hat{h}_{i j}^{l}$ denotes the estimate of $h_{i j}^{l}$, and $\eta_{n}^{0}, \eta_{n}^{1}$ denote the combined ISI and noise. The Alamouti algorithm was originally developed for flat fading channels and so does not take into consideration the ISI introduced by frequency-selective fading channels. Some modifications have to be made to in order to combat ISI and obtain multipath diversity gain. Recall the received signals at antenna rx0 are

$$
\begin{aligned}
r_{n}^{0} & =h_{00}^{2} s_{n-1}^{0}-h_{00}^{1} s_{n-1}^{1 *}+\underline{h_{00}^{0} s_{n}^{0}}+\ldots+w_{n}^{0} ; \\
r_{n}^{1} & =-h_{00}^{2} s_{n-1}^{1 *}+\underline{\underline{h_{00}^{1} s_{n}^{0}}}+\ldots+h_{10}^{1} s_{n}^{1}+\underline{h_{10}^{0} s_{n}^{0 *}+w_{n}^{1} ;} \\
r_{n+1}^{0} & =\underline{\underline{\underline{h_{00}^{2} s_{n}^{0}}}}-h_{00}^{1} s_{n}^{1 *}+\ldots+\underline{\underline{h_{10}^{1} s_{n}^{0 *}}}+h_{10}^{0} s_{n+1}^{1}+w_{n+1}^{0} \\
r_{n+1}^{1} & =-h_{00}^{2} s_{n}^{1 *}+\ldots+\underline{\underline{\underline{h_{10}^{2} s_{n}^{0 *}}}}+h_{10}^{1} s_{n+1}^{1}+\ldots+w_{n+1}^{1} .
\end{aligned}
$$

One can see from these equations that the desired symbols $s_{n}^{0}, s_{n}^{1}$ not only appear in the first-tap terms (with one line underneath), but also appear in the second-tap terms (with two lines underneath), as well as in the thirdtap terms (with three lines underneath). Statistically, the first tap is the strongest tap in the SUI-3 channel (meaning $\mathrm{E}\left[\left|h_{i j}^{0}\right|^{2}\right]>\mathrm{E}\left[\left|h_{i j}^{1}\right|^{2}\right]$ and $\left.\mathrm{E}\left[\left|h_{i j}^{0}\right|^{2}\right]>\mathrm{E}\left[\left|h_{i j}^{2}\right|^{2}\right]\right)$. However, it is a quasi-static channel, channel coefficients change from block to block. For some channel realizations, the other taps can be stronger than the first one. Therefore, instead of always choosing the first tap as shown in (2), the Alamouti scheme can be applied on the strongest tap based on the channel estimates for a specific channel realization. Furthermore, in order to take advantage of multipath propagation and obtain diversity gain, we should apply the Alamouti scheme on the two strongest or all the three taps and combine the desired signals from different taps. The modified Alamouti algorithm based on three-path combining can be expressed as

$$
\begin{aligned}
\widetilde{s_{n}^{0}} & =\hat{h}_{00}^{0 *} r_{n}^{0}+\hat{h}_{10}^{0} r_{n}^{1 *}+\hat{h}_{00}^{1 *} r_{n}^{1}+\hat{h}_{10}^{1} r_{n+1}^{0 *}+\hat{h}_{00}^{2 *} r_{n+1}^{0}+\hat{h}_{10}^{2} r_{n+1}^{1 *} \\
& =\sum_{i, l} \hat{h}_{i 0}^{l *} h_{i 0}^{l} s_{n}^{0}+\epsilon_{n}^{0}=P s_{n}^{0}+\epsilon_{n}^{0} ; \\
\widetilde{s_{n}^{1}} & =\hat{h}_{10}^{0 *} r_{n}^{0}-\hat{h}_{00}^{0} r_{n}^{1 *}+\hat{h}_{10}^{1 *} r_{n}^{1}-\hat{h}_{00}^{1} r_{n+1}^{0 *}+\hat{h}_{10}^{2 *} r_{n+1}^{0}-\hat{h}_{00}^{2} r_{n+1}^{1 *} \\
& =\sum_{i, l} \hat{h}_{i 0}^{l *} h_{i 0}^{l} s_{n}^{1}+\epsilon_{n}^{1},
\end{aligned}
$$

where $P=\sum_{i, l} \hat{h}_{i 0}^{l *} h_{i 0}^{l}$ is the total received power from different paths, and $\epsilon_{n}^{0}, \epsilon_{n}^{1}$ can be approximated as complex Gaussian random variables with zero mean and variance
$N_{\epsilon}$

$$
\begin{aligned}
N_{\epsilon} & =\left(\left|h_{00}^{0}\right|^{2}+\left|h_{10}^{0}\right|^{2}\right)\left(\left|h_{00}^{1}\right|^{2}+\left|h_{10}^{1}\right|^{2}+\left|h_{00}^{2}\right|^{2}+\left|h_{10}^{2}\right|^{2}+N_{0}\right) \\
& +\left(\left|h_{00}^{1}\right|^{2}+\left|h_{10}^{1}\right|^{2}\right)\left(\left|h_{00}^{0}\right|^{2}+\left|h_{10}^{0}\right|^{2}+\left|h_{00}^{2}\right|^{2}+\left|h_{10}^{2}\right|^{2}+N_{0}\right) \\
& +\left(\left|h_{00}^{2}\right|^{2}+\left|h_{10}^{2}\right|^{2}\right)\left(\left|h_{00}^{0}\right|^{2}+\left|h_{10}^{0}\right|^{2}+\left|h_{00}^{1}\right|^{2}+\left|h_{10}^{1}\right|^{2}+N_{0}\right)
\end{aligned}
$$

The conditional PDFs of $\widetilde{s_{n}^{0}}$ and $\widetilde{s_{n}^{1}}$ are thus derived as

$$
\begin{aligned}
& f\left(\widetilde{s_{n}^{0}} \mid s_{m}\right)=\frac{1}{\pi N_{\epsilon}} \exp \left(-\frac{\widetilde{\mid s_{n}^{0}}-\left.P s_{m}\right|^{2}}{N_{\epsilon}}\right) ; \\
& f\left(\widetilde{s_{n}^{1}} \mid s_{m}\right)=\frac{1}{\pi N_{\epsilon}} \exp \left(-\frac{\widetilde{\mid s_{n}^{1}}-\left.P s_{m}\right|^{2}}{N_{\epsilon}}\right) .
\end{aligned}
$$

For the QPSK modulated signals, the symbol LLR $\lambda\left(s_{n}\right)=\lambda\left(x_{n}\right)+j \lambda\left(y_{n}\right)$ to bits LLRs $\lambda\left(u_{n}^{\prime}[0]\right), \lambda\left(u_{n}^{\prime}[1]\right)$ mapping rule is simply $\lambda\left(u_{n}^{\prime}[0] ; O\right)=\lambda\left(x_{n}\right) ; \quad \lambda\left(u_{n}^{\prime}[1] ; O\right)=$ $\lambda\left(y_{n}\right)$. Next, we show how the LLR value of $x_{n}$ and $y_{n}$ can be derived from $\widetilde{s_{n}^{0}}$. The LLRs $\lambda\left(u_{n+1}^{\prime}[0]\right), \lambda\left(u_{n+1}^{\prime}[1]\right)$ can be computed similarly based on $\widetilde{s_{n}^{1}}$. Based on (5), the LLR value of $x_{n}$ can be computed as

$\lambda\left(x_{n}\right)=\ln \frac{\left.f\left(\widetilde{s_{n}^{0}} \mid s_{0}\right)+f \widetilde{\left(s_{n}^{0}\right.} \mid s_{3}\right)}{f\left(\widetilde{s_{n}^{0}} \mid s_{1}\right)+f\left(\widetilde{s_{n}^{0}} \mid s_{2}\right)} \approx \ln \frac{\exp \left(-\widetilde{s_{n}^{0}}-\left.P s_{+}\right|^{2} / N_{\epsilon}\right)}{\exp \left(-\left|\widetilde{s_{n}^{0}}-P s_{-}\right|^{2} / N_{\epsilon}\right)}$

$$
=\frac{1}{N_{\epsilon}}\left\{\widetilde{s_{n}^{0}}-\left.P s_{-}\right|^{2}-\widetilde{\mid s_{n}^{0}}-\left.P s_{+}\right|^{2}\right\}=\frac{2 P}{N_{\epsilon}} \operatorname{Re}\left\{s_{+}^{*} \widetilde{s_{n}^{0}}-s_{-}^{*} \widetilde{s_{n}^{0}}\right\},
$$

where $s_{+}$denotes the QPSK symbol corresponding to $\max \left\{f\left(\widetilde{s_{n}^{0}} \mid s_{0}\right), f\left(\widetilde{s_{n}^{0}} \mid s_{3}\right)\right\}$, and $s_{-}$denotes the QPSK symbol corresponding to $\max \left\{f\left(\widetilde{s_{n}^{0}} \mid s_{1}\right), f\left(\widetilde{s_{n}^{0}} \mid s_{2}\right)\right\}$ since the real part of the symbols $s_{0}, s_{3}$ corresponds to 0 , and the real part of the symbols $s_{1}, s_{2}$ corresponds to 1 . Dual maxima rule [20] is used in (6) utilizing the fact that one term usually dominates each sum. Similarly,

$$
\lambda\left(y_{n}\right)=\ln \frac{f\left(\widetilde{s_{n}^{0}} \mid s_{0}\right)+f\left(\widetilde{s_{n}^{0}} \mid s_{1}\right)}{f\left(\widetilde{s_{n}^{0}} \mid s_{2}\right)+f\left(\widetilde{s_{n}^{0}} \mid s_{3}\right)} \approx \frac{2 P}{N_{\epsilon}} \operatorname{Re}\left\{s_{+}^{*} \widetilde{s_{n}^{0}}-s_{-}^{*} \widetilde{s_{n}^{0}}\right\},
$$

where $s_{+}$denotes the QPSK symbol corresponding to $\max \left\{f\left(\widetilde{s_{n}^{0}} \mid s_{0}\right), f\left(\widetilde{s_{n}^{0}} \mid s_{1}\right)\right\}$, and $s_{-}$denotes the QPSK symbol corresponding to $\max \left\{f\left(\widetilde{s_{n}^{0}} \mid s_{2}\right), f\left(\widetilde{s_{n}^{0}} \mid s_{3}\right)\right\}$ since the imaginary part of the symbols $s_{0}, s_{1}$ corresponds to 0 , and the imaginary part of the symbols $s_{2}, s_{3}$ corresponds to 1 .

\section{B. Equalizer design at the subsequent cancellation stages}


The summation in (4) is carried out over all possible values of $i \in\{0,1\}$, and $l \in\{0,1,2\}$. One can see that this modified scheme also leads to temporal diversity gain in addition to the spatial diversity gain obtained by the original Alamouti scheme expressed in (2). On the other hand, however, $\epsilon_{n}^{0}, \epsilon_{n}^{1}$ in (4) also contain more ISI terms compared to $\eta_{n}^{0}, \eta_{n}^{1}$ in (2), which in turn will have a detrimental effect on the overall system performance. To tackle this problem, we can employ the multistage interference cancellation technique to cancel the contribution of the ISI terms. Let us denote $\bar{s}_{n-i}^{0}, \bar{s}_{n-i}^{1}$ as a soft estimate of $s_{n-i}^{0}, s_{n-i}^{1}$ from previous stage $\left(\left\{\bar{s}_{n-i}=\bar{x}_{n-i}+j \bar{y}_{n-i}\right\}\right.$ is computed according to its LLR value as $\bar{s}_{n-i}=\tanh \left[\lambda\left(x_{n-i}\right) / 2\right] / \sqrt{2}+$ $\left.j \tanh \left[\lambda\left(y_{n-i}\right) / 2\right] / \sqrt{2}\right)$. To simplify the notation, the iteration (stage) index is omitted whenever no ambiguity arises. Given a channel estimate $\hat{h}_{i j}^{l}$ and symbol estimates $\left\{\bar{s}_{n-i}^{0}, \bar{s}_{n-i}^{1}\right\}$, the ISI canceled version of the received signal $r_{n}^{0}$, denoted as $\bar{r}_{n}^{0}$ can be written according to (1) as

$$
\begin{aligned}
& \bar{r}_{n}^{0}=\left(h_{00}^{2} s_{n-1}^{0}-\hat{h}_{00}^{2} \bar{s}_{n-1}^{0}\right)-\left(h_{00}^{1} s_{n-1}^{1 *}-\hat{h}_{00}^{1} \bar{s}_{n-1}^{1 *}\right)+\underline{h_{00}^{0} s_{n}^{0}} \\
& +\left(h_{10}^{2} s_{n-1}^{1}-\hat{h}_{10}^{2} \bar{s}_{n-1}^{1}\right)+\left(h_{10}^{1} s_{n-1}^{0 *}-\hat{h}_{10}^{1} \bar{s}_{n-1}^{0 *}\right)+\underline{h_{10}^{0} s_{n}^{1}}+w_{n}^{0} .
\end{aligned}
$$

Other ISI canceled versions of the received signals, e.g., $\bar{r}_{n}^{1}, \bar{r}_{n+1}^{0}, \bar{r}_{n+1}^{1}$ can be formed similarly, i.e., by canceling the contribution from the symbols other than $s_{n}^{0}, s_{n}^{1}$. Using the aforementioned combining technique, the soft decisions of $s_{n}^{0}, s_{n}^{1}$ can now be formed based upon the ISI canceled signals as

$$
\begin{aligned}
\widetilde{s_{n}^{0}} & =\hat{h}_{00}^{0 *} \bar{r}_{n}^{0}+\hat{h}_{10}^{0} \bar{r}_{n}^{1 *}+\hat{h}_{00}^{1 *} \bar{r}_{n}^{1}+\hat{h}_{10}^{1} \bar{r}_{n+1}^{0 *}+\hat{h}_{00}^{2 *} \bar{r}_{n+1}^{0}+\hat{h}_{10}^{2} \bar{r}_{n+1}^{1 *} \\
& =\sum_{i, l} \hat{h}_{i 0}^{l *} h_{i 0}^{l} s_{n}^{0}+\varepsilon_{n}^{0}=P s_{n}^{0}+\varepsilon_{n}^{0} ;
\end{aligned}
$$$$
\widetilde{s_{n}^{1}}=\ldots=\sum_{i, l} \hat{h}_{i 0}^{l *} h_{i 0}^{l} s_{n}^{1}+\varepsilon_{n}^{1}
$$

where $P=\sum_{i, l} \hat{h}_{i 0}^{l *} h_{i 0}^{l}$ is the total received power from different paths, and $\varepsilon_{n}^{0}, \varepsilon_{n}^{1}$ denote the noise plus cancellation residual. Given correct decision feedback, all the ISI terms will be eliminated. The variance of $\varepsilon_{n}^{0}, \varepsilon_{n}^{1}$ will be much smaller than that of $\epsilon_{n}^{0} \epsilon_{n}^{1}$ in (4) and $\eta_{n}^{0} \eta_{n}^{1}$ in (2), consequently, the BER performance will be greatly improved. The combined noise and cancellation residual $\varepsilon_{n}^{0}, \varepsilon_{n}^{1}$ can be approximated as a Gaussian random variable with zero mean and variance $N_{\varepsilon}$, i.e., $\varepsilon_{n} \sim \mathcal{N}\left(0, N_{\varepsilon}\right)$. In the case of perfect cancellation, $N_{\varepsilon}=P N_{0}$. The conditional PDF is thus derived as $f\left(\widetilde{s_{n}^{0}} \mid s_{m}\right)=\frac{1}{\pi N_{\varepsilon}} \exp \left(-\frac{\left|\widetilde{s_{n}^{0}}-P s_{m}\right|^{2}}{N_{\varepsilon}}\right)$. The LLRs $\lambda\left(x_{n}\right), \lambda\left(y_{n}\right)$ can be derived in the same way as (7) in Section III. At the beginning of the iterative process, the symbol estimates $\left\{\bar{s}_{n-i}^{0}, \bar{s}_{n-i}^{1}\right\}$ needed for interference cancellation are derived by the modified Alamouti (the noncancellation) algorithm expressed by (4). In the following stages, they are obtained from the output of the Log-MAP decoder as depicted in Fig. 2.

\section{MMSE BASEd TURBo EQUALIZATION}

For comparison purpose, we briefly present the linear MMSE filter based turbo equalization proposed in [10,11].
The original algorithm is modified to suit the QPSK constellation and extended to the STBC coded FWA systems considered in this paper.

First, we reform equation (3) in vector form as $\mathbf{r}_{n}=$ $\mathbf{H s}_{n}+\mathbf{w}_{n}$ where $\mathbf{r}_{n}=\left[\begin{array}{llll}r_{n}^{0} & r_{n}^{1} & r_{n+1}^{0} & r_{n+1}^{1}\end{array}\right]^{T}, \quad \mathbf{w}_{n}=$ $\left[\begin{array}{llll}w_{n}^{0} & w_{n}^{1} & w_{n+1}^{0} & w_{n+1}^{1}\end{array}\right]^{T}$, and $\mathbf{s}_{n}=\left[\begin{array}{lll}s_{n-1}^{0} & -s_{n-1}^{1 *} & s_{n}^{0}-\end{array}\right.$

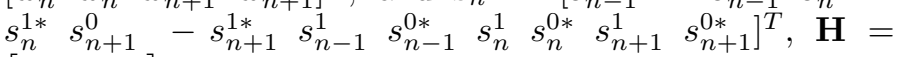
$\left[\begin{array}{ll}\mathbf{H}_{0} & \mathbf{H}_{1}\end{array}\right]$, where

$$
\begin{aligned}
\mathbf{H}_{0} & =\left[\begin{array}{cccccc}
h_{00}^{2} & h_{00}^{1} & h_{00}^{0} & 0 & 0 & 0 \\
0 & h_{00}^{2} & h_{00}^{1} & h_{00}^{0} & 0 & 0 \\
0 & 0 & h_{00}^{2} & h_{00}^{1} & h_{00}^{0} & 0 \\
0 & 0 & 0 & h_{00}^{2} & h_{00}^{1} & h_{00}^{0}
\end{array}\right] \\
\mathbf{H}_{1} & =\left[\begin{array}{cccccc}
h_{10}^{2} & h_{10}^{1} & h_{10}^{0} & 0 & 0 & 0 \\
0 & h_{10}^{2} & h_{10}^{1} & h_{10}^{0} & 0 & 0 \\
0 & 0 & h_{10}^{2} & h_{10}^{1} & h_{10}^{0} & 0 \\
0 & 0 & 0 & h_{10}^{2} & h_{10}^{1} & h_{10}^{0}
\end{array}\right] .
\end{aligned}
$$

The interference canceled vectors are formed as

$$
\begin{aligned}
& \mathbf{r}_{n}^{0 \prime}=\mathbf{r}_{n}-\mathbf{H} \tilde{\mathbf{s}}_{n}^{0}=\mathbf{H}\left[\mathbf{s}_{n}-\tilde{\mathbf{s}}_{n}^{0}\right]+\mathbf{w}_{n} ; \\
& \mathbf{r}_{n}^{1 \prime}=\mathbf{r}_{n}-\mathbf{H} \tilde{\mathbf{s}}_{n}^{1}=\mathbf{H}\left[\mathbf{s}_{n}-\tilde{\mathbf{s}}_{n}^{1}\right]+\mathbf{w}_{n},
\end{aligned}
$$

where $\tilde{\mathbf{s}}_{n}^{0}=\left[\begin{array}{llllll}\bar{s}_{n-1}^{0} & -\bar{s}_{n-1}^{1 *} & 0 & -\bar{s}_{n}^{1 *} & \bar{s}_{n+1}^{0} \quad-\end{array}\right.$ $\left.\begin{array}{lllllll}\bar{s}_{n+1}^{1 *} & \bar{s}_{n-1}^{1} & \bar{s}_{n-1}^{0 *} & \bar{s}_{n}^{1} & 0 & \bar{s}_{n+1}^{1} & \bar{s}_{n+1}^{0 *}\end{array}\right]^{T} ; \quad \tilde{\mathbf{s}}_{n}^{1}=\left[\begin{array}{llll}\bar{s}_{n-1}^{0} & -\end{array}\right.$

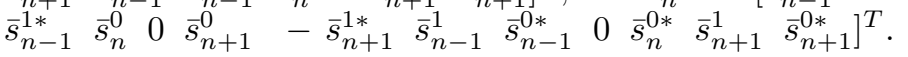
They contain the soft estimate of the interference symbols from the previous iteration. Like the proposed scheme, the MMSE equalization algorithm removes the effect of ISI by applying soft interference cancellation as shown in (10). Linear MMSE filters are applied on the ISI canceled vectors $\mathbf{r}_{n}^{0 \prime}, \mathbf{r}_{n}^{1 \prime}$ to obtain $z_{n}^{0}=\mathbf{c}_{n}^{0 *} \mathbf{r}_{n}^{0 \prime}, z_{n}^{1}=\mathbf{c}_{n}^{1 *} \mathbf{r}_{n}^{1 \prime}$, where the filter coefficients vectors, $\mathbf{c}_{n}^{0}$ and $\mathbf{c}_{n}^{1}$ are chosen to minimize the mean square error. We use $\mathbf{c}_{n}^{0}$ as an example, it is derived as $\mathbf{c}_{n}^{0}=\arg \min \mathrm{E}\left\{\left(s_{n}-\mathbf{c}_{n}^{0 *} \mathbf{r}_{n}^{0 \prime}\right)^{2}\right\}=\mathbf{R}^{-1} \mathbf{p}^{*}$ where

$$
\begin{aligned}
\mathbf{R} & =\mathrm{E}\left\{\mathbf{r}_{n}^{0 /} \mathbf{r}_{n}^{0 / *}\right\}=\mathrm{E}\left\{\mathbf{H}\left[\mathbf{s}_{n}-\tilde{\mathbf{s}}_{n}^{0}\right]\left[\mathbf{s}_{n}-\tilde{\mathbf{s}}_{n}^{0}\right]^{*} \mathbf{H}^{*}\right\} \\
& =\mathbf{H} \nabla_{n} \mathbf{H}^{*}+N_{0} \mathbf{I} ; \\
\nabla_{n} & =\mathrm{E}\left\{\left[\mathbf{s}_{n}-\tilde{\mathbf{s}}_{n}^{0}\right]\left[\mathbf{s}_{n}-\tilde{\mathbf{s}}_{n}^{0}\right]^{*} \mathbf{H}^{*}\right\} \\
& =\operatorname{diag}\left\{\ldots, 1-\left|\bar{s}_{n-1}^{1}\right|^{2}, 1, \ldots, 1-\left|\bar{s}_{n}^{1}\right|^{2}, 1, \ldots\right\} ; \\
\mathbf{p} & =\mathrm{E}\left\{\mathbf{r}_{n}^{0 / *} s_{n}^{0}\right\}=\mathrm{E}\left\{\left(\mathbf{H}\left[\mathbf{s}_{n}-\tilde{\mathbf{s}}_{n}^{0}\right]\right)^{*} s_{n}^{0}\right\} \\
& =\mathrm{E}\left\{\left[\begin{array}{c}
h_{00}^{0} s_{n}^{0} \\
h_{00}^{1} s_{n}^{0}+h_{10}^{0} s_{n}^{0 *} \\
h_{00}^{2} s_{n}^{0}+h_{10}^{1} s_{n}^{0 *} \\
h_{10}^{2} s_{n}^{0 *}
\end{array}\right] s_{n}^{0}\right\}=\left[\begin{array}{llll}
h_{00}^{0 *} & h_{00}^{1 *} & h_{00}^{2 *} & 0
\end{array}\right] .
\end{aligned}
$$

The vector $\mathbf{p}$ in (11) is derived based on the fact that $\mathrm{E}\left[s_{m} s_{m}^{*}\right]=1, \mathrm{E}\left[s_{m} s_{m}\right]=0$ for QPSK modulation. Obviously, direct implementation of the MMSE equalization on this STBC coded system leads to diversity loss (the terms $h_{10}^{0 *}, h_{10}^{1 *}, h_{10}^{2 *}$ disappear from the vector $\left.\mathbf{p}\right)$. The filter output $z_{n}$ is approximated as a Gaussian random variable with mean value $\mu$, i.e., $z_{n}=\mu s_{n}+\gamma$. Conditioned on the modulated symbol $s_{n}$, the mean and variance can be computed as

$$
\begin{aligned}
\mu & =\mathrm{E}\left[z_{n} s_{n}^{*}\right]=\mathbf{c}_{n}^{0 *} \mathrm{E}\left[\mathbf{r}_{n}^{0 \prime} s_{n}^{*}\right]=\mathbf{c}_{n}^{0 *} \mathbf{H} \mathrm{E}\left[\left(\mathbf{s}_{n}-\tilde{\mathbf{s}}_{n}^{0}\right) s_{n}^{*}\right]=\mathbf{c}_{n}^{0 *} \mathbf{p}_{n}^{0 *} \\
N_{\gamma} & =\mathrm{E}\left[\left|z_{n}\right|^{2}\right]-\mu^{2}=\mathrm{E}\left[\mathbf{c}_{n}^{*} \mathbf{r}_{n}^{\prime} \mathbf{r}_{n}^{\prime *} \mathbf{c}_{n}\right]-\mu^{2}=\mathbf{c}_{n}^{*} \mathrm{E}\left[\mathbf{r}_{n}^{\prime} \mathbf{r}_{n}^{\prime *}\right] \mathbf{c}_{n}-\mu^{2} \\
& =\mathbf{c}_{n}^{*} \mathbf{R} \mathbf{R}^{-1} \mathbf{p}_{n}^{*}-\mu^{2}=\mathbf{c}_{n}^{*} \mathbf{p}_{n}^{*}-\mu^{2}=\mu-\mu^{2}
\end{aligned}
$$


The conditional PDF can be expressed as $f\left(z_{n} \mid s_{n}=\right.$ $\left.s_{m}\right)=\frac{1}{\pi N_{\gamma}} \exp \left(-\frac{\left|z_{n}-\mu s_{m}\right|^{2}}{N_{\gamma}}\right)$. The LLRs $\lambda\left(x_{n}\right), \lambda\left(y_{n}\right)$ can be derived in the same way as (7) in the previous section.

\section{Numerical Results}

Numerical results are presented in this section to assess the performance of the proposed turbo equalization scheme. We employ a rate $1 / 3$ Maximum Free Distance convolutional code with constraint length 5 and generator polynomials $(25,33,37)$ in octal form. During each Monte-Carlo run, the block size is set to 2000 information bits followed by 4 tails bits to terminate the trellis, which corresponds to $2004 \times 3=6012$ coded bits or 3006 QPSK symbols, 200 of which are used as pilots to acquire a channel estimate $\hat{h}_{i j}^{l}$. Channel estimation is conducted with the modified maximum likelihood algorithm introduced in [21]. The coded bits are interleaved by a random interleaver. QPSK symbols are transmitted over the SUI-3 FWA MIMO channels. The channel coefficients vary from one block to another, however, they are assumed to remain constant during the transmission of one block of data. It is therefore a quasistatic channel. The antenna correlation coefficient is set to 0.4. The noise variance $N_{0}$ and path delays are assumed to be known to the receiver. The simulation curves are obtained by averaging the simulation results over at least 300 channel realizations. To study the behavior of each algorithm, the number of iterations is set to 4 since it is observed that less than 4 stages (in addition to the noncancellation stage described in Section III) are needed for all the discussed schemes to converge.

Fig. 3 shows the comparison of different equalization schemes for the 2TX-1RX STBC FWA system. The proposed turbo equalizer is investigated using both the ML channel estimates and the perfect channel state information (CSI). We use the first modified version of the Alamouti algorithm (see equation (4)) for the initial stage of the turbo equalization. At the following stages, the second modified version of the Alamouti algorithm (see (8) and (9)) is employed to cancel ISI using decision feedback from the previous iteration. Compared to the results achieved assuming perfect CSI, the performance loss due to the channel estimation errors is within a fraction of $1 \mathrm{~dB}$. As expected, the proposed scheme performs much better than the MMSE based turbo equalization due to the reason that the proposed scheme not only preserves the features of the original Alamouti algorithm, i.e., achieving spatial diversity with simple linear processing at the receiver, but also takes advantage of the multipath propagation and obtains the temporal diversity by multipath combining and reduces the effect of ISI by interference cancellation. On the contrary, the MMSE scheme cannot utilize the spatial diversity as discussed in Section IV. To work around this problem, the solution in [13] is not to use STBC. In our case, we can transmit the same symbols $\left\{s_{n}^{0}, s_{n}^{1}\right\}$ from two transmit antennas instead of applying space-time coding and transmit $\left\{s_{n}^{0},-s_{n}^{1 *}\right\}$ from the first antenna and transmit $\left\{s_{n}^{1}, s_{n}^{0 *}\right\}$ from the second antenna. In this way, the elements of the $\mathbf{p}$ vector in (11) will consist of the channel gains from both channels, e.g., $\mathbf{p}=\left[\begin{array}{lll}h_{00}^{0 *}+h_{10}^{0 *} & h_{00}^{1 *}+h_{10}^{1 *} & h_{00}^{2 *}+h_{10}^{2 *}\end{array}\right]$, and spatial diversity can be obtained. On the other hand, how-

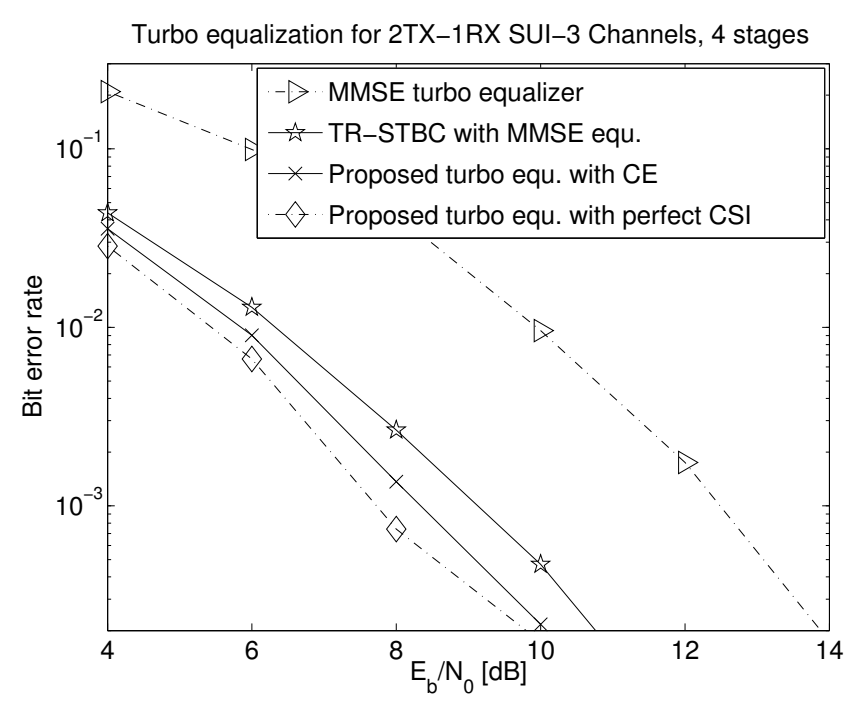

Figure 3: Comparison of different turbo equalization schemes for the 2TX-1RX STBC FWA system. The curves represent the 4 th stage turbo equalization excluding the first non-cancellation stage. The modified Alamouti scheme uses 3-path combining.

ever, the MMSE algorithm shown in Section IV requires matrix inversion at symbol rate; whereas the proposed scheme only requires linear processing as the original Alamouti algorithm. Therefore, it greatly reduces the complexity compared to the MMSE based turbo equalization.

In Fig. 3, the proposed scheme is also compared with the TR-STBC which is a transmit diversity scheme specially designed for frequency selective channels [18]. The TR-STBC itself only decouples the symbol streams from two transmit antennas. It, however, does not resolve the ISI in each symbol stream. The ISI of course still has to be handled by an equalizer. To this end, we apply a 7-tap MMSE equalizer in the TR-STBC system after the symbol streams are decoupled. As shown in Fig. 3, the proposed turbo equalization scheme outperform the STBC scheme with MMSE equalizer. Equalizers with length greater than 7 are tested for the TR-STBC system and are shown to have very marginal gain compared to 7 -tap equalizer.

The proposed scheme in Fig. 3 is implemented with 3path combining. Different combining (selection) strategies are compared in Fig. 4 for the 2TX-2RX STBC FWA system. Direct implementation of the Alamouti scheme on the first path (1-path non-selective) or the strongest path (1path selective) yields the worst results. We also see that the selective scheme performs better than the non-selective one. Off all the discussed strategies, the 2-path and 3-path combining schemes are much superior to the 1-path schemes, especially, the 3-path scheme has the best performance. Obviously, in order to fully achieve the temporal diversity from the multipath propagation, we need to combine the signals from all the paths.

\section{Conclusions}

In this paper, we design a low complexity turbo equalizer for space-time block coded FWA systems based on the Alamouti algorithm, which has been modified in such way that 


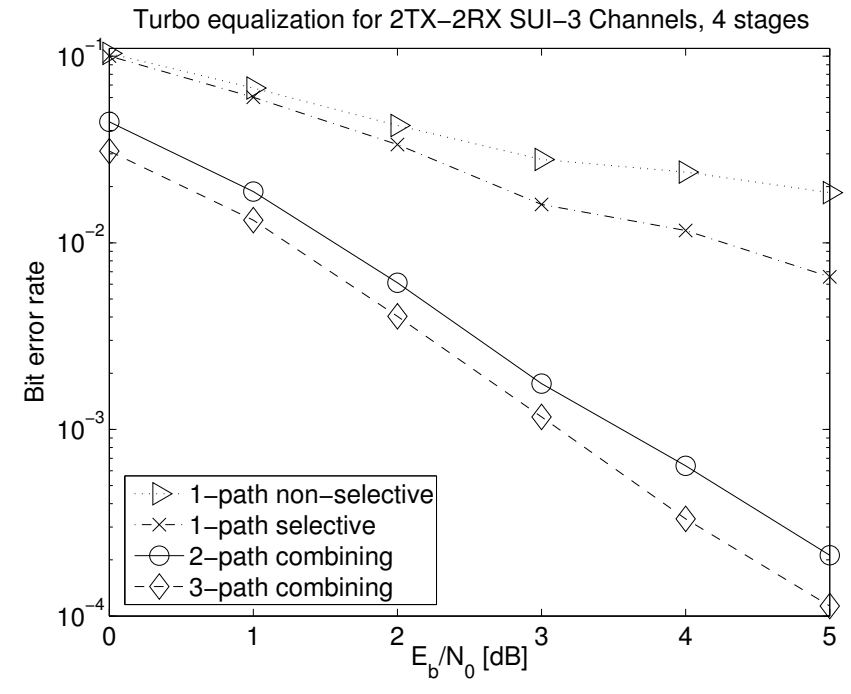

Figure 4: Comparison of different modified Alamouti schemes for the 2TX-2RX STBC FWA system. The curves represent the 4 th stage turbo equalization excluding the first non-cancellation stage.

both spatial and temporal diversities are exploited and the effect of ISI is mitigated. We also show that although the conventional MMSE filter-based turbo equalizer achieves ISI mitigation, it, however, fails to exploit the spatial diversity if implemented directly to the STBC systems. Therefore, the proposed scheme significantly outperforms the conventional MMSE scheme with a reduced complexity. Results also show that the proposed scheme yields superior performance to the TR-STBC transmit diversity scheme for frequency selective channels. Although originally designed for the FWA MIMO channels, the proposed turbo equalization scheme can be applied to other static or quasi-static frequency selective fading channels.

\section{REFERENCES}

[1] H. Bolcskei, A. Paulraj, K. Hari, R. Nabar, W. Lu. "Fixed Broadband Wireless Access: State of the Art, Challenges, and Future Directions". IEEE Communication Magazine, pp. 100-108, Jan. 2001.

[2] IEEE 802.16 Working Group on Broadband Wireless Access Standards. available at http://grouper.ieee.org/groups/802/16/. 2002.

[3] Draft ETSI TS 101, Broadband Radio Access Networks (BRAN), HIPERMAN. available at http://portal.etsi.org/bran/Summary.asp, 2002.

[4] C. Eklund. "IEEE Standard 802.16: a technical overview of the wirelessMAN air interface for broadband wireless access". IEEE Communications Magazine, pp. 98-107, June, 2002.

[5] V. Tarokh, N. Seshadri, A. Calderbank. "Space-time codes for high data rates wireless communications: performance criterion and code construction". IEEE Transactions on Information Theory, vol. 44, pp. 744-765, March 1998.

[6] A. Alamouti. "A simple transmit diversity technqiue for wireless communications". IEEE Journal on Selected Areas in Communications, vol. 16, no. 8, pp. 1451-1458, Oct. 1998.
[7] V. Tarokh, N. Seshadri, A. Calderbank. "Space-time block coding from orthogonal designs". IEEE Transactions on Information Theory, vol. 45, pp. 1456-1467, July 1999.

[8] S. Sandhu, A. Paulraj. "Space-time block codes versus spacetime trellis codes", Proceedings IEEE Internation Conference on Communications, vol. 4, pp. 1132-1136, 2001.

[9] C. Douillard. "Iterative correction of intersymbol interference: turbo-equalization". European Transactions on Telecommunications, pp. 507-511, Sept. 1995.

[10] X. Wang, H. Poor. "Iterative (turbo) soft interference cancellation and decoding for coded CDMA". IEEE Transactions on Communications, vol. 47, pp. 1046-1061, July 1999.

[11] M. Tuchler, R. Koetter, A. Singer. "Turbo equalization: principles and new results". IEEE Transactions on Communications, vol. 50, pp. 754-767, May 2002.

[12] G. Bauch, N. Al-Dhahir. "Reduced-complexity space-time turbo equalization for frequency-selective MIMO channels". IEEE Transactions on Wireless Communications, vol. 1, no. 40, pp. 819-828, Oct. 2002.

[13] X. Wautelet, A. Dejonghe, L. Vandendorpe. "MMSEbased fractional turbo receiver for space-time BICM over frequency-selective MIMO fading channels". IEEE Transactions on Signal Processing, vol. 52, no. 6, pp. 1804-1809, June 2004.

[14] T. Abe, T. Matsumoto. "Space-time turbo equalization in frequency-selective MIMO channels". IEEE Transactions on Vehicular Technology, vol. 52, no. 3, pp. 469-482, May 2003.

[15] B. Lu and X. Wang. "Iterative receivers for multiuser spacetime coding systems". IEEE Journals on Selected Areas in Communications, vol. 18, no. 11. pp. 2322-2335, Nov. 2000.

[16] S. Jayaweera, H. Poor. "A rake-based iterative receiver for space-time block-coded multipath CDMA". IEEE Transactions on Signal Processing, vol. 52, no. 3. pp. 796-806, March 2004.

[17] N. Veselinovic, T. Matsumoto, M. Juntti. "Iterative MIMO turbo multiuser detection and equalization for STTrC-coded systems with unknown interference". EURASIP Journal on Wireless Communications and Networking, no. 2, pp. 309321, Dec. 2004.

[18] E. Lindskog, A. Paulraj. "A transmit diversity scheme for channels with intersymbol interference". IEEE International Conference on Communications, pp. 307-311, June 2002.

[19] V. Erceg. "An empirically based path loss model for wireless channels in suburban environments". IEEE JSAC, vol. 17, no. 7, pp. 1205-1211, July 1999.

[20] A. Viterbi. "An intuitive justification and a simplified implementation of the MAP decoder for convolutional codes". IEEE Journals on Selected Areas in Communications, vol. 16, no. 2, pp. 260-264, Feb. 1998.

[21] P. Xiao, R. Carrasco, I. Wassell. "Estimation of FWA MIMO channels". Proceedings IEEE Information Theory Workshop, ITW'2006. To appear. 